\title{
Traduire
}

Une autre perspective sur r tr traduction

Revue française de la traduction

$235 \mid 2016$

Luxe, mode... et traduction !

\section{Pour une bonne collaboration avec les agences de traduction}

\section{Alina Cincan}

Traducteur : Olivier den Hartigh

\section{OpenEdition \\ Journals}

Édition électronique

URL : https://journals.openedition.org/traduire/862

DOI : $10.4000 /$ traduire.862

ISSN : 2272-9992

\section{Éditeur}

Société française des traducteurs

Édition imprimée

Date de publication : 1 décembre 2016

Pagination : 94-102

ISSN : 0395-773X

Référence électronique

Alina Cincan, «Pour une bonne collaboration avec les agences de traduction », Traduire [En ligne], 235 |

2016, mis en ligne le 01 décembre 2018, consulté le 05 juillet 2021. URL : http://

journals.openedition.org/traduire/862 ; DOI : https://doi.org/10.4000/traduire.862 


\section{Pour une bonne collaboration avec les agences de traduction}

\section{Alina Cincan}

Cet article est signé d'une directrice d'agence de traduction ayant son siège au Royaume-Uni. Nous tenons à signaler que nos colonnes sont également ouvertes aux auteurs souhaitant s'exprimer sur le thème des relations avec les clients directs.

Invitée en 2015 à intervenir lors d'une Conférence sur la traduction et la localisation, j'ai choisi d'aborder le thème de "Travailler avec les agences de traduction : avantages pour le client et pour le traducteur ". Deux raisons principales ont dicté ce choix : premièrement, parce que je voulais démontrer que les agences ne sont pas toujours le vilain petit canard du monde de la traduction comme certains voudraient le faire croire, même s'il existe de mauvaises agences, tout comme il existe de mauvais traducteurs, de mauvais comptables, de mauvais avocats, etc. Deuxièmement, parce que $72 \%$ des traducteurs consultés lors de l'élaboration de cet exposé souhaitaient mieux comprendre le fonctionnement des agences : le traitement réservé à leurs candidatures, à leur CV, le choix du prestataire, etc.

Cet article vise donc à reprendre l'essentiel de l'exposé présenté devant la Conférence. Aux traducteurs et traductrices professionnels d'en tirer les enseignements.

\section{Fonctionnement d'une agence de traduction}

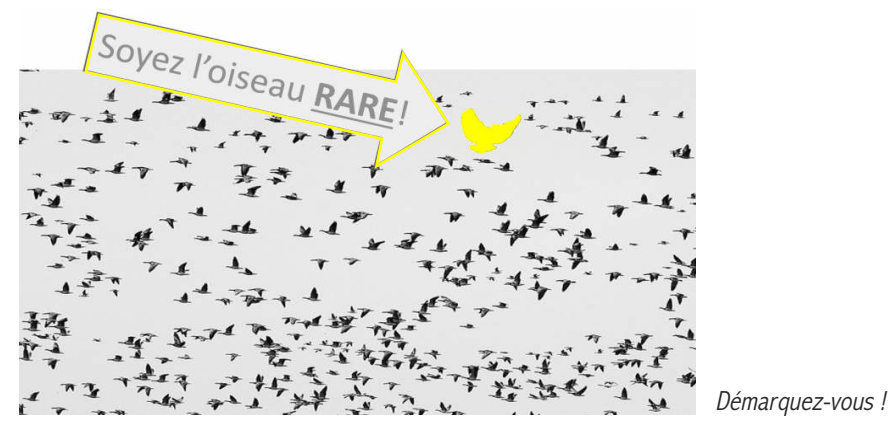




\section{L'importance de la touche personnelle}

Tout commence avec l'envoi d'une candidature. Il s'agit du premier contact entre le traducteur et l'agence. Nous connaissons tous les dictons "c'est la première impression qui compte ", ou mieux encore " on a une seule chance de faire une bonne première impression ", deux formules qui, bien que galvaudées, n'en restent pas moins pertinentes. II faut savoir que des études ont montré qu'une opinion se forge généralement en sept secondes chrono. D'où la nécessité d'être l'oiseau bleu de l'illustration, et de sortir du lot. Pour vous faire remarquer, il y a donc des actions à préconiser et des écueils à éviter.

Au cours de votre carrière, vous avez dû recevoir au moins une fois un courriel commençant par la petite phrase "Cher traducteur ", "Cher collègue " ou, pire encore : "Cher prestataire". Et je suis prête à parier que vous avez réagi de façon négative, consignant le message en question à la corbeille sans envisager une seule seconde d'y répondre. Rien d'étonnant alors, si vous envoyez à une agence un message qui commence par " M. le gestionnaire de projet", "M. le Directeur des ressources humaines ", "Madame, Monsieur ", etc., que votre email ait toutes les chances de subir le même sort. Pourquoi? Parce que des centaines d'emails de ce type sont reçus chaque jour, chaque semaine.

Comment donc vous assurer que le vôtre reçoive toute l'attention qu'il mérite ? En l'occurrence, il vous suffira de quelques minutes pour trouver le nom des personnes à la tête de l'agence. Une démarche personnalisée qui témoignera de votre désir de travailler avec elles (et non pas avec la première agence qui daignera répondre) et qui, à coup sûr, vous mettra en situation favorable. Pour ce qui nous concerne, moins de $4 \%$ des courriels de candidature que nous recevons sont nominatifs, ce qui les fait automatiquement sortir du lot. II va sans dire que de tels messages font toujours l'objet d'une réponse de notre part.

Une fois rédigé votre courriel personnalisé, il vous faudra appliquer la règle dite " du père Noël ". La règle du père Noël, kézako me direz-vous? Eh bien, si le père Noël trouve le temps de vérifier deux fois d'affilée sa liste de cadeaux pour chacun de plusieurs milliards de bambins, vous devez vous aussi prendre le temps de vérifier votre message, et pas seulement au moment de postuler, bien entendu.

Croyez-moi sur parole, car depuis le jour où j'ai appelé quelqu'un "Booby(1) " au lieu de "Bobby(2) " (j'en rougis encore), je puis vous assurer que je vérifie, revérifie et re-revérifie mes messages écrits.

(1) NDT : idiot, crétin en anglais.

(2) NDT : un des diminutifs du prénom Robert. 


\section{Le respect des consignes}

Par là j'entends que, si vous cherchez à remettre votre candidature ou à vous inscrire en tant que traducteur auprès d'une agence, vous avez tout intérêt à prendre quelques minutes pour vous familiariser avec les modalités d'inscription ou de candidature de l'agence en question.

L'envoi d'un courriel convient-il ? L'agence dispose-t-elle d'un formulaire à remplir ? En suivant les procédures préconisées, vous aurez la certitude que :

1. Votre candidature parviendra au bon destinataire.

2. Tout risque de perte de temps (le sien et le vôtre) sera évité.

\section{Références - mode d'emploi}

Comme de nombreuses agences de traduction, nous demandons aux candidats de nous fournir des références. Un conseil primordial à ce propos : avant de transmettre les coordonnées de personnes susceptibles d'appuyer votre candidature, veillez à obtenir leur accord et à les tenir au courant de votre démarche. Attention : certaines entreprises ont pour principe de ne jamais donner de référence. Or, sans ces références, votre candidature sera rejetée d'office. Et celui dont les coordonnées sont transmises à son insu risque de très mal prendre la chose.

\section{Tirer le meilleur parti de votre CV}

Le CV est un élément essentiel de votre candidature. Un CV bien rédigé mettra l'accent sur vos compétences et vous permettra, encore une fois, de vous démarquer de la concurrence. Cela dit, en quoi consiste un bon $\mathrm{CV}(3)$ ? Voilà une question délicate digne d'un exposé à elle toute seule. Toutefois, il y a quelques règles générales à respecter :

Langues de travail : la première information dont a besoin une agence de traduction concerne vos langues de travail. Aussi, ne gardez pas cette indication pour la fin de votre CV (à mon avis, le format Europass n'est pas idéal pour le traducteur). Mettez vos langues source et cible clairement en évidence, inscrivez-les tout en haut de votre CV, voire carrément dans le nom du fichier.

II importe également d'adapter votre CV en fonction de votre statut de traducteur débutant ou expérimenté. Un CV doit mettre en valeur. II ne doit pas vous desservir mais vous aider dans votre démarche.

(3) Voir e-book gratuit de Marta Stelmaszak consacré aux CV et axé sur le monde de la traduction : http://wantwords.co.uk/school/lesson-57-you-need-a-cv-that-works-ebook-on-cv-writing-in-translation-2/ 
Les traducteurs débutants se plaignent du paradoxe du chien qui se mord la queue. Jeunes diplômés (ou personnes ayant peu d'expérience), on ne leur confie pas suffisamment de traductions pour leur permettre d'acquérir l'expérience nécessaire. Face à une telle situation, comment peuvent-ils faire pour gagner leurs galons et comment devraient-ils se mettre en valeur dans leur CV ?

Pour le débutant, il existe différentes façons de peaufiner son art : faire de la traduction bénévole (le choix du bon projet est un tout autre débat) ou suivre des cours dans le cadre de la formation professionnelle continue (FPC) (formations en ligne, par exemple). De telles initiatives permettent au néophyte de manifester son envie d'exercer cette merveilleuse profession. Vous pourrez également lancer un blog qui fera office de vitrine et mettra en avant vos qualités rédactionnelles (une belle plume étant le propre de tout traducteur qui se respecte), votre savoir et votre passion pour ce métier.

Quant au traducteur expérimenté, son atout majeur est forcément son expérience. En effet, l'expérience et les domaines de spécialisation sont les deux volets essentiels de l'argumentaire de vente d'un professionnel chevronné.

Autre observation importante à propos du CV : il doit être succinct et efficace à la fois. Au moment de mon intervention en 2015 , le record du CV le plus long était détenu par un document de seize pages. J'espère d'ailleurs que vous êtes bien assis, car ce record fut battu peu de temps après par un CV de... 86 pages, ni plus, ni moins, qui devait (probablement) énumérer tous les projets entrepris par son auteur. Mais plus inquiétant encore, il y figurait également une liste de 52 clients avec leurs noms et leurs coordonnées, très vraisemblablement sans l'autorisation des personnes concernées. Qui va prendre la peine de lire un tel pavé ? Je vous conseille donc de vous restreindre à deux pages maximum. Vous pouvez, bien entendu, citer des projets sur lesquels vous avez travaillé, mais cantonnez-vous aux plus importants ou aux plus pertinents. Et, de grâce, ne mettez pas les coordonnées de vos clients dans votre CV.

En matière de mise en forme, il convient de privilégier le format PDF, et ce pour deux raisons :

- II permettra de préserver le formatage et la présentation (contrairement aux fichiers Word qui, en raison des nombreuses versions MS Office, peuvent perdre leur mise en forme et renvoyer une image parfois chaotique à l'écran).

- Avec un fichier PDF, les fraudeurs auront plus de mal à vous voler votre CV, voire votre identité. D'après le Translator Scammers Directory, près de $4000 \mathrm{CV}$ faux ou volés circuleraient actuellement sur la toile (sans parler de ceux dont nous n'avons pas encore connaissance). Alors, évitez d'offrir votre CV sur un plateau aux fraudeurs !

Enfin, dernier élément mais non des moindres, n'oubliez pas d'appliquer, comme toujours, la règle du père Noël. 


\section{L'adhésion à une association professionnelle : un atout}

Les traducteurs que j'ai consultés étaient également nombreux à s'interroger sur l'utilité d'une adhésion à une association professionnelle. J'y répondrai par l'affirmative.

Plus précisément, je dirais que le seul inconvénient de l'adhésion à une association est peutêtre l'aspect financier. Ceci étant, les avantages d'un tel investissement sont nombreux. Permettez-moi d'en citer deux, et non des moindres. Premier avantage : votre qualité de membre d'une association de traducteurs témoigne de votre engagement professionnel. II s'agit bien de votre métier et non pas d'une activité complémentaire (ce qui correspond à l'une des définitions du terme " professionnel „ figurant dans le dictionnaire). Second avantage, qui revêt également une très grande importance, la plupart de ces associations organisent des événements, des conférences et des formations FPC qui sont autant d'occasions d'épanouissement professionnel.

Pour ce qui est de l'association sur laquelle jeter son dévolu, force est de reconnaître que les traducteurs et interprètes n'ont que l'embarras du choix. Comment sélectionner ? II va sans dire que votre choix dépendra de votre lieu de résidence ou de celui de la plupart de vos clients (directs ou indirects). Au Royaume-Uni, mentionnons le CloL (Chartered Institute of Linguists) et I'ITI (Institute of Translation \& Interpreting). En tant qu'association internationale, largement reconnue et respectée, I'IAPTI (International Association of Professional Translators and Interpreters) mérite également une mention spéciale. En France, l'association la plus connue est probablement la SFT (Société française des traducteurs).

Parmi les traducteurs dans mon échantillon, certains ont parlé des portails internet. Même si y créer un profil n'est pas forcément une mauvaise idée, il ne faudra jamais oublier que ce sont de simples plateformes d'offre, à ne pas confondre avec des associations professionnelles. Ces portails facilitent cependant le réseautage, l'échange et l'entraide entre traducteurs, la recherche d'une clientèle...

Quant à l'attribution des marchés de traduction, une question revient sans cesse : quel est le facteur déterminant dans le choix d'un traducteur : ses diplômes ? Son expérience ? Son adhésion à une association? Ses tarifs ? Ses domaines de spécialisation? Pour paraphraser une boutade bien connue des traducteurs, je répondrais que tout dépend du contexte... (ou du projet)!

\section{Travailler AVEC et non pas POUR les agences de traduction}

Une autre étude menée auprès des traducteurs m'a permis de cerner les raisons pour lesquelles certain(e)s rechignent à travailler avec les agences (voir l'illustration ci-après). 


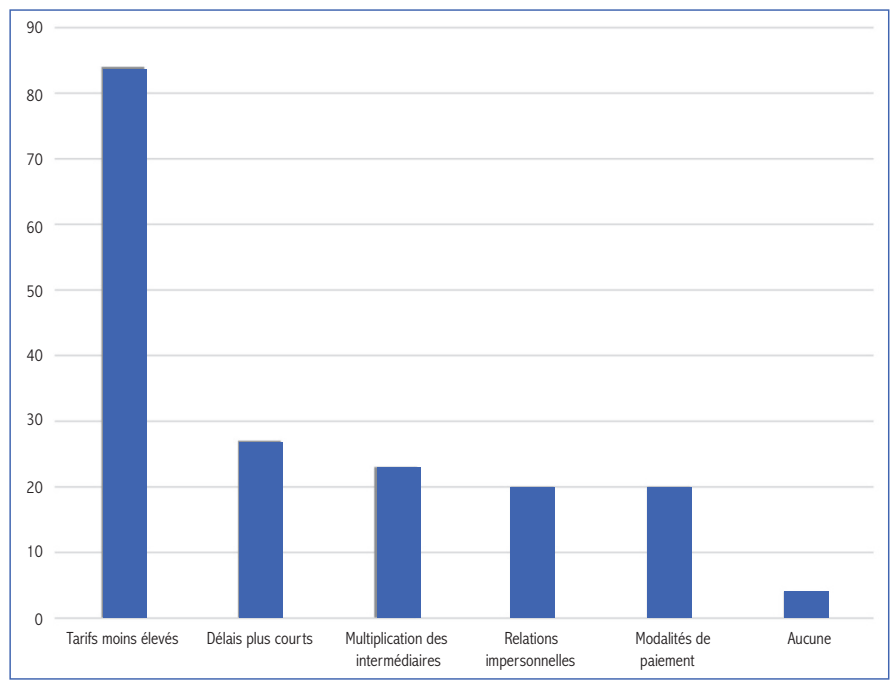

Mon constat est le suivant : si vous acceptez des tarifs qui ne vous enchantent pas, des délais que vous avez du mal à respecter, des relations interpersonnelles qui ne vous plaisent pas, des modalités de paiement qui ne vous satisfont pas, alors vous travaillez POUR l'agence de traduction.

Pour changer la donne et travailler AVEC une agence, vous devez comprendre qu'il s'agit d'une relation commerciale, avec deux parties prenantes, et que ces deux parties sont sur un pied d'égalité. Elles doivent agir en partenariat. Aucune des deux parties n'est obligée d'accepter en bloc les desiderata de l'autre.

Ce qui m'amène à l'importance d'un contrat écrit, que ce soit sous la forme d'un bon de commande ou d'un contrat-cadre (peu de traducteurs nous ont demandé jusqu'ici de signer leurs conditions générales). À ma connaissance, certaines associations, dont l'ITI, disposent d'un modèle de contrat standard pour traducteurs.

\section{Collaboration avec une agence de traduction : les avantages}

\section{Côté traducteur}

L'enquête qui a mis en lumière les inconvénients d'une collaboration avec les agences de traduction a aussi mis en exergue certains avantages : flux de travail réguliers, professionnalisme (le traducteur n'a pas besoin d'expliquer au client ce qu'est un outil de TAO ou une 


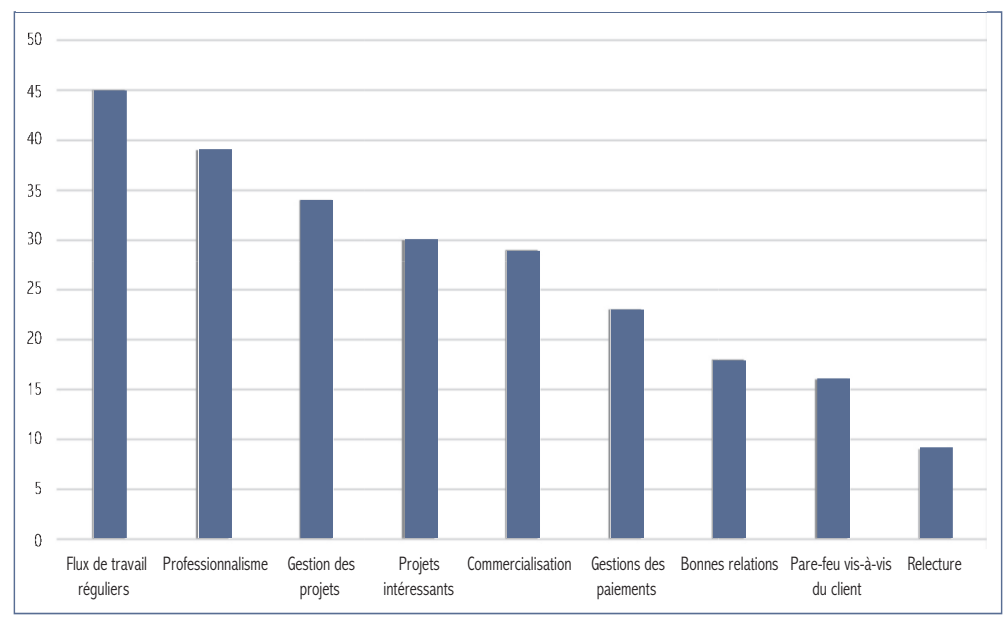

mémoire de traduction), gestion des projets, projets intéressants, commercialisation (ce qui permet au traducteur de se consacrer à son véritable métier), gestion des paiements, bonnes relations, pare-feu vis-à-vis du client, relecture.

Il est intéressant de noter que les aspects financiers et relationnels figurent autant parmi les avantages que parmi les inconvénients. II faut donc croire que les indépendants pour lesquels ce sont des avantages ont dû trouver une agence avec laquelle ils jouissent d'une bonne collaboration. Tout traducteur qui le veut pourra trouver une bonne agence de traduction. II suffit de se renseigner autour de soi et auprès des nombreux forums et groupes axés sur ce sujet et de procéder à des négociations en cas de besoin (si l'une ou l'autre des parties n'accepte pas la totalité des modalités imposées par l'autre).

\section{Côté client}

Pour ce qui est du client final, les avantages sont éventuellement plus évidents :

\section{- Sélection minutieuse des traducteurs}

Rappelons que quelque 4000 CV contrefaits ou volés circuleraient sur le marché, chose qu'ignore très probablement le client final. Selon João Roque Dias(4) tel qu'il l'a exposé lors de la conférence de l'ATA en 2014, $14 \%$ des agences de traduction ne seraient pas au courant

(4) Traducteur portugais et auteur du "Translator Scammers Directory " : http://www.translator-scammers.com/translator-scammers-directory.htm 
de cette affaire : quid alors du non-professionnel, qui aura nécessairement plus de mal à trier le bon grain de l'ivraie ? Laisser aux agences de traduction le soin de se charger de cet aspect paraîtrait donc judicieux.

Le choix du bon traducteur par rapport à un projet n'est pas toujours évident. Un client qui travaille dans un seul et même domaine, dans une seule paire linguistique, a certainement intérêt à collaborer en prise directe avec un traducteur indépendant. Mais celui qui doit faire traduire un ensemble de contrats, de supports marketing, de manuels techniques, etc. trouvera peut-être plus commode de confier à une agence le soin de sélectionner des traducteurs adéquats.

\section{- Projets multilingues, délais serrés ou projets volumineux}

Là encore, ce sont des cas de figure où le client pourrait bénéficier de l'intervention d'une agence de traduction, étant donné le caractère chronophage de tels projets et la bonne coordination requise.

Dernier point (et non des moindres) : comment démontrer votre qualité de traducteur professionnel ?

Comment se définit un traducteur professionnel ? Question complexe, aux multiples facettes. J'ai abordé ce thème en octobre 2015 dans le cadre d'un événement organisé par la Chartered Institute of Linguists(5), et en juin 2016 au Portugal à l'invitation de l'APTRAD (l'association portugaise de traducteurs et d'interprètes)(6). Dans cet article, je me cantonnerai à un seul aspect, à savoir votre comportement. Que ce soit en direct ou sur internet, l'image que vous transmettrez aura forcément une incidence (positive ou négative) sur votre activité de traducteur. Méfiez-vous donc de vos propos relatifs aux clients et aux collègues : ils pourraient un jour vous desservir.

En conclusion, j'insisterai sur la nécessité d'un professionnalisme à tous les niveaux (à commencer par votre email de présentation, par votre CV et par votre capacité à vous distinguer de la masse...).

Vous avez tout à gagner en collaborant pleinement avec une agence plutôt qu'en instaurant une simple relation de travail avec elle. L'agence pourra mettre à profit les bons rapports qui en résulteront ; le client final aussi.

(5) http://www.ciol.org.uk

(6) Associação Portuguesa de Tradutores e Intérpretes : http://www.aptrad.pt 
Que vos partenaires soient des agences de traduction ou des clients directs, vous devrez toujours faire preuve d'un professionnalisme sans faille. En conjuguant nos forces pour prêcher la bonne parole et tirer la qualité des traductions vers le haut, nous ne pourrons qu'aboutir à une meilleure image pour tous les acteurs de la traduction.

alina@inboxtranslation.com

Nota : Article publié en allemand par le BDÜ(7), en anglais par I'ITI(8) et en néerlandais par la CBTI-BKVT(9).

Traduction française par Olivier den Hartigh, réadaptée par Christine Cross.

Traductrice expérimentée depuis plus de dix ans, Alina Cincan est membre du Chartered Institute of Linguists et modératrice à l'Institute of Translation \& Interpreting au Royaume-Uni. Elle est par ailleurs directrice d'Inbox Translation (http://inboxtranslation.com/). Elle intervient dans le cadre d'événements sectoriels en vue de partager son expérience et de promouvoir le recours aux bonnes pratiques dans le domaine de la traduction.

(7) http://www.bdue-fachverlag.de/fachverlag/detail_fachzeitschrift/66, journal MDÜ de décembre 2015.

(8) The Institute of Translation and Interpreting (http://www.iti.org.uk), ITI Bulletin mai-juin 2016.

(9) CBTI-BKVT - Belgian Chamber of Translators and Interpreters (http://www.cbti-bkvt.org) à paraître dans " De Taalkundige/Le Linguiste " en langue néerlandaise fin 2016. 